\title{
The Y-stenting technique for bifurcation stenosis and bioprosthetic valve frame fracture prior to valve-in-valve transcatheter pulmonary valve replacement in a child
}

\author{
Michał Gałeczka ${ }^{1}$, Sebastian Smerdziński ${ }^{1}$, Wojciech Sadowski ${ }^{2}$, Marcin Demkow ${ }^{3}$, Jacek Białkowski ${ }^{1}$, \\ Roland Fiszer ${ }^{1}$ \\ ${ }^{1}$ Department of Congenital Heart Defects and Paediatric Cardiology, FMS in Zabrze, Medical University of Silesia in Katowice, \\ Silesian Centre for Heart Diseases, Zabrze, Poland \\ ${ }^{2}$ Biocybernetics Laboratory, Prof. Z. Religa Foundation of Cardiac Surgery Development, Zabrze, Poland \\ ${ }^{3}$ Department of Coronary Artery and Structural Heart Diseases, Institute of Cardiology, Warsaw, Poland
}

Adv Interv Cardiol 2020; 16, 2 (60): 206-208

DOI: https://doi.org/10.5114/aic.2020.96066

\section{Introduction}

Tetralogy of Fallot (TOF) corrective surgery, especially using a transannular patch, carries a high risk of reintervention [1]. Surgical pulmonary valve replacement with a bioprosthetic valve (BPV) is a well-established method of treating postoperative right outflow tract dysfunction [2]. Since BPV function deteriorates and patient-prosthesis mismatch increases with growth, reintervention is necessary. To avoid the reoperation risk, valve-in-valve (ViV) transcatheter pulmonary valve replacement (TPVR) has become an attractive alternative [3]. The BPV true inner diameter (ID) is shorter than the nominal BPV size by $2 \mathrm{~mm}$ or more, which limits the ViV therapy [4]. The valve implanted within the BPV makes the effective lumen even narrower. Since the first report by Tanase et al. [5], intentional fracture of the BPV frame using ultra-high-pressure balloons has become a valuable option to optimize the hemodynamic outcomes of ViV in the BPV in a pulmonic position [6].

\section{Case report}

The case concerns a 17-year-old, 69 kg, asymptomatic boy after a TOF complete repair with a transannular patch. The patient was re-operated at the age of 10 with a stentless valve and only 1 year later with a $23 \mathrm{~mm}$ Carpentier-Edwards Perimount Magna bioprosthetic valve (Edwards Lifesciences Inc., Irvine, USA). Pulmonary stenosis (PS) with maximum/mean gradient of 100/55 mm Hg, moderate pulmonary regurgitation, and dilated right ventricle were established during transthoracic echocardi- ography. Computed tomography (CT) revealed a $12 \mathrm{~mm}$ supravalvular PS and proximal narrowing of pulmonary arteries: right (RPA) to 9 and left (LPA) to $6 \mathrm{~mm}$ (Figure $1 \mathrm{~A}$ ).

In order to test transcatheter treatment options, a 3D CT-derived silicone model of the aforementioned region with an embedded valve was prepared. Subsequently, the Y-stenting technique for bifurcation stenosis was selected. This includes implantation of a long hybrid-cell designed stent into the supravalvular PS and LPA and another stent into the RPA through the struts of the first stent (Figure 1 B). Moreover, an experimental bench test with cracking of the $23 \mathrm{~mm}$ Magna BPV using a $24 \mathrm{~mm}$ Atlas balloon (Bard Peripheral Vascular Inc., Tempe, USA) was performed. The lower restrictive frame of the valve cracked at $14 \mathrm{~atm}$. A preprocedural CT scan excluded risk of coronary artery compression in the intended valve landing zone. Finally, the patient was qualified for ViV TPVR and informed consent was obtained.

A PS gradient of $51 \mathrm{~mm} \mathrm{Hg}$, right-to-left ventricle pressure ratio (RV/LV) of 0.73 , and a BPV ID of $19 \mathrm{~mm}$ were confirmed by catheterization. A $48 \mathrm{~mm}$ AndraStent XXL (AndraMed GmbH, Reutlingen, Germany) was implanted into the LPA, pulmonary trunk, and Magna valve on a $22 \mathrm{~mm}$ BIB balloon (NuMED Inc., NY, USA). The supravalvular diameter increased to 18.3 and the LPA origin increased to $16 \mathrm{~mm}$. The next step was postponed for a month to enable partial stent ingrowth. After pre-dilatation with a $12 \mathrm{~mm}$ Atlas Gold balloon, a $21 \mathrm{~mm}$ AndraStent XXL on a $16 \mathrm{~mm}$ MaxiLD (Cordis, Florida, USA) balloon was implanted into the RPA through a semi-opened first stent scaffold. The RPA expanded to

\section{Corresponding author:}

Michal Galeczka MD, Department of Congenital Heart Defects and Paediatric Cardiology, FMS, Medical University of Silesia,

Silesian Centre for Heart Diseases, 9 M. Curie-Sklodowskiej St, 41-800 Zabrze, Poland, phone: +4832373 3669 ,

e-mail: michalgaleczka@gmail.com

Received: 27.11.2019, accepted: 24.02.2020. 

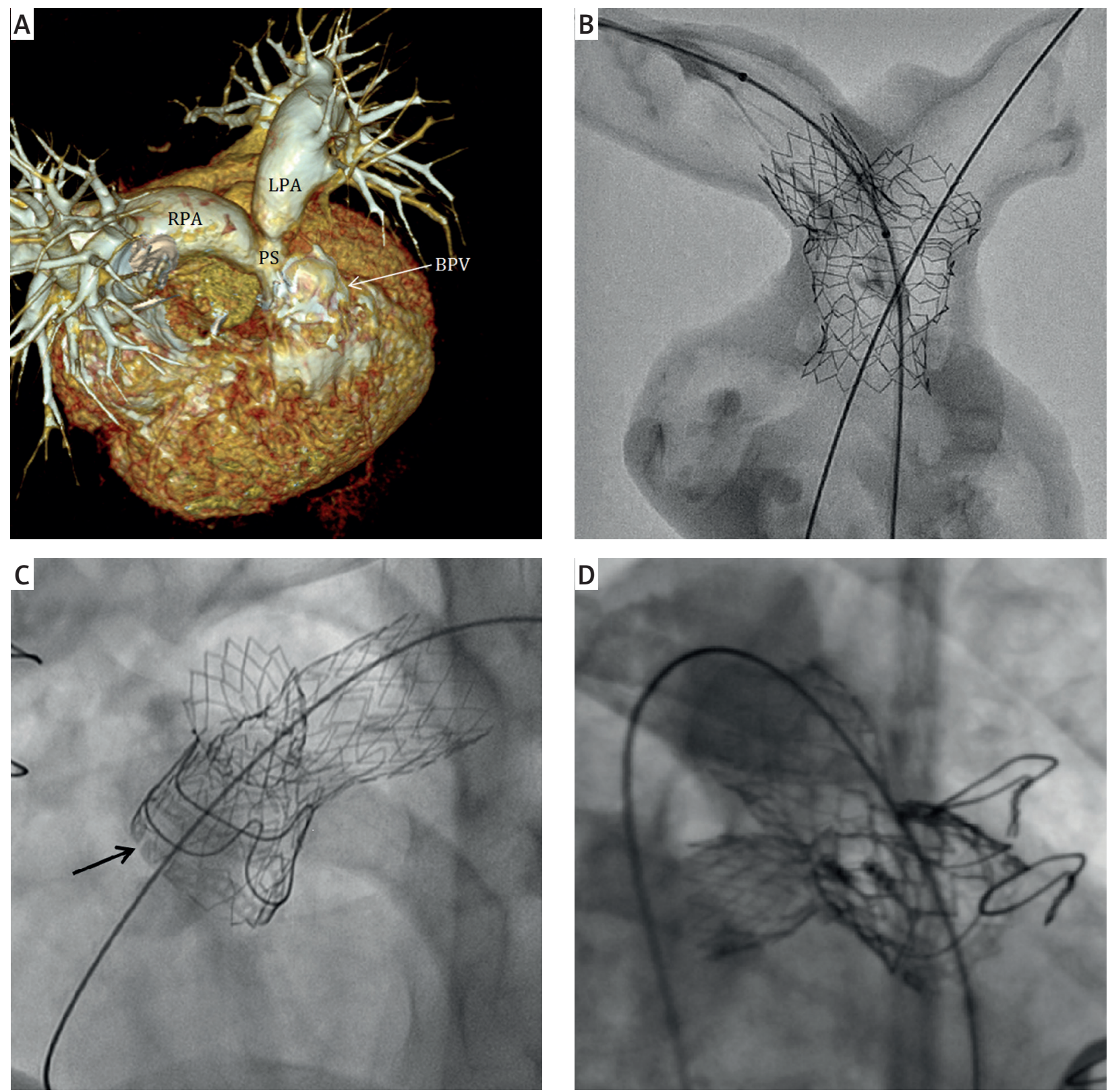

Figure 1. A - Computed tomography of a 17-year-old tetralogy of Fallot patient after bioprosthetic Edwards Magna valve (BPV) surgical replacement. Supravalvular pulmonary (PS) and bifurcation stenosis. B - Three-dimensional computed tomography-derived silicone model of the right ventricle outflow tract with supravalvular (PS) and bifurcation stenosis: $48 \mathrm{~mm}$ AndraStent XXL implanted into PS and LPA and through this stent's cells a $21 \mathrm{~mm}$ AndraStent XXL was implanted in the RPA. C - $48 \mathrm{~mm}$ AndraStent XXL implanted into both PS and LPA and $21 \mathrm{~mm}$ AndraStent XXL implanted into RPA. Lower frame of $23 \mathrm{~mm}$ Magna valve cracked at 18 atm with $24 \mathrm{~mm}$ Atlas balloon, arrow - frame separation. D - $23 \mathrm{~mm}$ Edwards Sapien XT valve implanted in the upper part of a $23 \mathrm{~mm}$ Magna valve

$R P A$ - right pulmonary artery, LPA - left pulmonary artery.

$11.4 \mathrm{~mm}$. Magna valve fracture was performed 3 months later with a $24 \mathrm{~mm}$ Atlas balloon. The lower BPV frame cracked at $18 \mathrm{~atm}$ and ID increased to $22 \mathrm{~mm}$ (Figure $1 \mathrm{C}$ ). A $23 \mathrm{~mm}$ Edwards Sapien XT valve was subsequently implanted slightly above the Magna valve (balloon overfilled with $3 \mathrm{ml}$ ) (Figure $1 \mathrm{D}$ ). The XT valve overdilation allowed the BPV ID to be expanded to 24 , the XT valve ID to 22, and supravalvular stenosis to $21 \mathrm{~mm}$. The residual gradient decreased to $30 \mathrm{~mm} \mathrm{Hg}$ with an RV/LV ratio of 0.39 and persisted at the supravalvular stenosis (top of the XT valve). There were no procedure-related complications during any stage. Life-long aspirin, 6 months of clopidogrel, and continuous infective endocarditis prophylaxis were administered. In a 10-month follow-up, 
the patient is asymptomatic with a mean PS gradient $<25 \mathrm{~mm} \mathrm{Hg}$ and no PR in routine control echocardiography.

\section{Discussion}

BPV has a four-component structure: tissue valve, supporting frame, sewing ring, and polymer cloth covering. ViV tactics in BPVs cause an unacceptable limitation of the eventual lumen. Furthermore, a high residual gradient after TPVR brings a greater risk for valve failure, death [7], and infective endocarditis [8].

Depending on the frame material, BPVs can be either fractured or stretched. The ultra-high-pressure Kevlar balloons are non-compliant and resistant to overdilation with a nominal burst pressure of $18 \mathrm{~atm}$; however, successful inflation up to $26 \mathrm{~atm}$ was reported [6]. According to the literature, the balloon should be minimally $1-2 \mathrm{~mm}$ larger than the true ID of the BPV [6]. BPV fracture proved to be safe in both in vitro and in vivo studies due to the surrounding Dacron sewing ring, which prevents vessel damage [5, 6, 9].

The complex bifurcation anatomy in our patient forced the non-standard procedure. A Y-stenting technique was selected in order to dilate the PS and pulmonary arteries. The long hybrid-cell stent was implanted into the PS and the LPA to enable efficient RPA dilation. However, staged rehabilitation of the obstructed right ventricle-to-pulmonary artery conduit with implantation of a stent on two balloons has also been described [10]. As cracking was performed with a $24 \mathrm{~mm}$ balloon, selection of a $23 \mathrm{~mm}$ Edwards XT valve seemed to be safe. The XT valve was implanted just below the bifurcation to dilate the narrowest part of the stent at a safe distance from coronary arteries. On the basis of the distance between coronary arteries and the pulmonic valve landing zone, the compression test was abandoned. The whole procedure was divided into stages to ensure proper stent stabilisation and to prevent displacement during the cracking of the BPV. In summary, supravalvular PS and bifurcation were significantly dilated and the gradient decreased to $30 \mathrm{~mm} \mathrm{Hg}$.

The Y-stenting technique for bifurcation stenosis and intentional fracture of the BPV frame with ultra-high-pressure balloon prior to ViV TPVR is possible in selected patients in order to prevent a hazardous reoperation.

\section{Conflict of interest}

Marcin Demkow is a proctor for Edwards Lifesciences Inc. The other authors declare no conflict.

\section{References}

1. Ylitalo P, Nieminen $H$, Pitkänen $O M$, et al. Need of transannular patch in tetralogy of Fallot surgery carries a higher risk of reoperation but has no impact on late survival: results of Fallot repair in Finland. Eur J Cardiothorac Surg 2015; 48: 91-7.
2. Nomoto R, Sleeper LA, Borisuk MJ, et al. Outcome and performance of bioprosthetic pulmonary valve replacement in patients with congenital heart disease. J Thorac Cardiovasc Surg 2016; 152: 1333-42.e3.

3. Cabalka AK, Asnes JD, Balzer DT, et al. Transcatheter pulmonary valve replacement using the melody valve for treatment of dysfunctional surgical bioprostheses: a multicenter study. J Thorac Cardiovasc Surg 2018; 155: 1712-24.e1.

4. Bapat V, Mydin I, Chadalavada S, et al. A guide to fluoroscopic identification and design of bioprosthetic valves: a reference for valve-in-valve procedure. Catheter Cardiovasc Interv 2013; 81: 853-61.

5. Tanase D, Grohmann J, Schubert S, et al. Cracking the ring of Edwards Perimount bioprosthesis with ultrahigh pressure balloons prior to transcatheter valve in valve implantation. Int J Cardiol 2014; 176: 1048-9.

6. Shahanavaz S, Asnes JD, Grohmann J, et al. Intentional fracture of bioprosthetic valve frames in patients undergoing valve-invalve transcatheter pulmonary valve replacement. Circ Cardiovasc Interv 2018; 11: e006453.

7. Georgiev S, Ewert P, Tanase D, et al. A low residual pressure gradient yields excellent long-term outcome after percutaneous pulmonary valve implantation. JACC Cardiovasc Interv 2019; 12: 1594-603.

8. Nordmeyer J, Ewert P, Gewillig M, et al. Acute and midterm outcomes of the post-approval MELODY Registry: a multicentre registry of transcatheter pulmonary valve implantation. Eur Heart J 2019; 40: 2255-64.

9. Allen KB, Chhatriwalla AK, Cohen DJ, et al. Bioprosthetic valve fracture to facilitate transcatheter valve-in-valve implantation. Ann Thorac Surg 2017; 104: 1501-8.

10. Moszura T, Surmacz R, Goreczny S, et al. Staged rehabilitation of obstructed right ventricle-to-pulmonary artery conduit with implantation of a Cheatham-Platinum stent mounted on two Tyshak-X balloons followed by a Melody valve. Adv Interv Cardiol 2017; 13: 182-3. 